\title{
Surface Regeneration of Gold-Coated Chip for Highly-Reproducible Surface Plasmon Resonance Immunoassays
}

\section{Chandra Kumar Dixit'1,2,3*}

${ }^{1}$ Department of Mechanical Engineering, Technion-Israel Institute of Technology, Israel

${ }^{2}$ Centre for Bioanalytical Sciences (CBAS), National Centre for Sensor Research, Dublin City University, Dublin 9, Ireland

${ }^{3}$ Applied Biochemistry Group, School of Biotechnology, Dublin City University, Dublin 9, Ireland

\begin{abstract}
We developed a simple procedure for the complete regeneration of silane-grafted gold surface. This method allowed the reuse of the regenerated gold chip for custom refunctionalization. In addition, we performed highly reproducible immunoassays using these chips over several cycles. The developed procedure was optimized and comprised of consecutive treatments of functionalized Au chip with $12 \mathrm{M} \mathrm{HCl}$ for 10 min and $29 \mathrm{~W}$ oxygen (O2)plasma for $5 \mathrm{~min}$. Monitoring and surface characterization of the developed methodology was performed with ellipsometry and Rutherford back scattering. The developed procedure was demonstrated on SPR-based Human Fetuin A (HFA) immunoassay, where the amino groups of APTES-functionalized Au chip were cross linked to the carboxyl groups of anti-HFA antibody using 1-ethyl-3-[3-dimethylaminopropyl] carbodiimide hydrochloride and sulfo$\mathrm{N}$-hydroxy succinimide. The APTES-functionalization, anti-HFA antibody immobilization and HFA binding on the regenerated SPR Au chip were highly reproducible over 40 HFA immunoassay cycles.
\end{abstract}

Keywords: Gold chip; APTES; Surface plasmon resonance; Surface functionalization; Human fetuin A immunoassay

\section{Introduction}

Regeneration of the surface immobilized with recognition biomolecules, such as antibody, after immunoassay is very important for expensive bioanalytical platforms such as Surface Plasmon Resonance (SPR), quartz crystal microbalance and micro-cantilevers. This makes the chips reusable thus making it cost-effective. Regeneration is usually done by breaking the antibody-antigen interactions using acidic or alkaline solution [1-4]. The strength of regeneration solution is directly proportional to the affinity of antibody towards its antigen and the stability of the antigen-antibody complex. The chemical composition and functional conformation of an antibody is affected by the ionic strength, $\mathrm{pH}$ and chemical nature of regeneration solution. The repeated exposure of an antibody to a strong regeneration solution adversely impacts its functional activity $[1,5,6]$. Therefore, a regeneration solution that does not affect the affinity and avidity of an antibody should be employed [1]. A wide range of regeneration solutions with varying regeneration efficiencies and effects on the functionality of immobilized antibodies had been reported [7-9]. The determination of an appropriate regeneration solution is a time-consuming and laborintensive process. However, although a regeneration solution leads to the complete regeneration of an antibody-bound surface, it decreases its activity after few weeks due to storage, contamination, biofouling, protein spreading and other complex effects. Such an antibody-bound SPR chip can no longer be employed for an immunoassay.

In this manuscript we are reporting a novel approach which relies on functionalization of Au surface with APTES [10] regenerating the surface of the used SPR chip rather than focussing on the regeneration of biomolecule interaction. In this new approach, regeneration was achieved by removing the adsorbed aminopropyl triethoxysilane (APTES) layer which will facilitate the complete removal of any immobilized biomolecules. This will tremendously increase the cost-effectiveness of any Au-coated SPR chip as the same chip can be reused over several cycles for performing the same or different immunoassays. The developed and optimized procedure involved consecutive treatments with hydrochloric acid ( $\mathrm{HCl})$ [11-14] and $\mathrm{O}_{2}$ plasma. The complete regeneration of Au-surface of SPR chip was monitored with ellipsometry and Rutherford Back Scattering (RBS). The increase in cost-effectiveness of SPR Au chip was determined by highly-reproducible silanization, anti-HFA antibody binding, and HFA detection in 40 consecutive immunoassay cycles on the same SPR chip after regeneration. The developed regeneration procedure would also be useful for many other biosensor formats that employ APTEScoated surfaces such as microcantilevers, quartz crystal microbalance and gold electrodes.

\section{Experimental Section}

\section{SPR Au chip preparation and silanization}

The Au chip was prepared following previously mentioned procedure reported by our group [15]. The assembled SPR Au chip was cleaned by treating with piranha solution $[3: 1(\mathrm{v} / \mathrm{v})$ ratio of $97.5 \%$ $\mathrm{H}_{2} \mathrm{SO}_{4}$ and $30 \% \mathrm{H}_{2} \mathrm{O}_{2}$ ] for three minutes followed by extensive DIW washing (at least five times) to remove the traces of piranha. The cleaned chip was then incubated with $2 \%(\mathrm{v} / \mathrm{v})$ APTES (in 30\% (v/v) ethanol) for $1 \mathrm{~h}$ at Room Temperature (RT) in a fume hood, which generated a SAM of silane.

\section{Regeneration studies}

The functionalized chips were subjected to acid-lysis of siloxane bonds [11-13] by $\mathrm{HCl}$. The acid-mediated lysis based regeneration was optimized using various HCL strengths $(5,10,12$ and $15 \mathrm{M})$, and exposure times $(5,10,20$ and $30 \mathrm{~min})$. The $\mathrm{O}_{2}$ plasma etching was also optimized at three different plasma powers viz. 12, 20 and $29 \mathrm{~W}$ at different exposure times of 3, 6, 9, 12 and $15 \mathrm{~min}$. Subsequently, the

*Corresponding author: Chandra Kumar Dixit, Department of Mechanical Engineering, Technion-Israel Institute of Technology, Israel, Tel: +972 54644 7363; E-mail: chandra.dixit2@mail.dcu.ie

Received March 26, 2014; Accepted April 28, 2014; Published May 05, 2014

Citation: Dixit CK (2014) Surface Regeneration of Gold-Coated Chip for HighlyReproducible Surface Plasmon Resonance Immunoassays. J Biosens Bioelectron 5: 149. doi: 10.4172/2155-6210.1000149

Copyright: (c) 2014 Dixit CK. This is an open-access article distributed under the terms of the Creative Commons Attribution License, which permits unrestricted use, distribution, and reproduction in any medium, provided the original author and source are credited. 
combined $\mathrm{HCl}$ and $\mathrm{O}_{2}$ plasma treatment procedure was optimized for $\mathrm{Au}$ surface regeneration.

\section{Ellipsometric analysis}

The thickness of the APTES layer on Au chip was measured using a Jobin Yvon Horiba UVISEL spectroscopic ellipsometer (Chilly Mazarin, France). The measurements of psi $(\psi)$ and delta $(\Delta)$ spectra were conducted at an incident angle of $70^{\circ}$ over a wavelength range of $350-1000 \mathrm{~nm}$. The instrument's inbuilt silicon dioxide dispersion layer fitting model was then followed to determine the effective thicknesses of Au on glass and APTES on Au.

\section{Rutherford backscattering (RBS) analysis}

The APTES-functionalized SPR Au chip was loaded into the scattering chamber that was maintained under high vacuum conditions. A collimated beam of $2 \mathrm{MeV} \mathrm{He}$ ions, generated by a $3.5 \mathrm{MV}$ HVEE Singletron accelerator, was normally incident onto the sample. The ions backscattered at $101.25^{\circ}$ scattering angle were collected and their energy was measured by an Ortec Ultra silicon solid-state detector. The resultant spectra were fitted using the SIMNRA software. The total Si areal density for each sample was extracted from the target structure that provides the best fit for the respective spectrum.

\section{SPR-based HFA immunoassay procedure}

Anti-HFA antibody ( $990 \mu \mathrm{L}$ of $100 \mu \mathrm{g} / \mathrm{mL}$ reconstituted in HBS) was incubated for $15 \mathrm{~min}$ at RT with $10 \mu \mathrm{L}$ of the cross-linking solution containing EDC $(4 \mathrm{mg} / \mathrm{mL})$ and sulfo-NHS $(11 \mathrm{mg} / \mathrm{mL})$ in $0.1 \mathrm{M}, \mathrm{pH}$ 4.7 MES buffer. Later, $100 \mu \mathrm{L}$ of the resulting EDC-sulfo-NHS activated antibody solution was flown over the silanized Au chip at $10 \mu \mathrm{L} / \mathrm{min}$ to prepare anti-HFA antibody-coated chip. The anti-HFA antibody immobilized chip was then blocked using sulfo-NHS acetate, which reacts to free amino groups on the surface. This was followed by BSAblocking in order to fill the void space on the chip thus, minimizing non-specific protein adsorption on the surface. Subsequently, the HFA at 0.6 to $20 \mathrm{ng} / \mathrm{mL}$ dilutions was assayed on the anti-HFA antibody immobilized chips $[15,16]$ for 40 cycles of regeneration.

Later, the SPR Au chip was regenerated by treatment with $12 \mathrm{M}$
$\mathrm{HCl}$ for $10 \mathrm{~min}$ followed with the $29 \mathrm{~W} \mathrm{O}_{2}$ plasma treatment for $5 \mathrm{~min}$. The regenerated $\mathrm{Au}$ chips were reused and analyzed for reproducible immobilization of anti-HFA antibody and HFA detection in consecutive runs for 40 cycles.

\section{Results and Discussion}

Various silane-based Au surface modification strategies have been employed for immunoassays; however, their use for SPR applications is fairly recent [16-18]. There are reports claiming the successful regeneration of thiol terminal silane-functionalized $\mathrm{Au}$ surfaces [19]. It was observed that strong acid treatment such as $\mathrm{HCl}$ can dissolve the siloxane bonds and remove upto the second APTES layer as the first APTES layer on the Au surface was very strongly bound through $\mathrm{Au}$-thiol interactions. However, there was no critical assessment of reusability and functional characterization of SPR Au chip. In addition, $\mathrm{HCl}$ may also affect the homogeneity of Au coating. In this report, we have developed a simple strategy, based on consecutive treatments with $\mathrm{HCl}$ and $\mathrm{O}_{2}$-plasma, for the complete regeneration of $\mathrm{Au}$ surface as illustrated in Figure 1. The regeneration studies were performed on the Au chips that were (a) functionalized with APTES, and (b) bound to anti-HFA antibody after APTES-functionalization. The thickness of APTES self-assembled monolayer (SAM) and the areal density of APTES on the bare, silanized and regenerated Au chip surfaces were determined by ellipsometry and RBS, respectively Figure 2 .

Twenty chips were APTES-functionalized and different regions on each chip (four spots in the corner and one spot in the centre) were analyzed to assess the homogeneity of silane layer. A SAM thickness of 5 $\mathrm{nm} \pm 1 \mathrm{~nm}$ was obtained in all the repeats. The thickness was calculated with the instrument software, which generates a differential reflection profile [designated as psi $(\psi)$ and delta $(\Delta)$ ] for each corresponding layer. The contact angle obtained for the SAM of APTES on Au surface was $59^{\circ} \pm 1^{\circ}$, which is consistent with previous findings [20]. The contact angle obtained for piranha-treated bare Au was $25^{\circ}$, which was in agreement with previous reports that claimed the contact angle of cleaned gold in the range of $0-30^{\circ}[21]$. The regeneration of Au surface by the developed procedure was confirmed by RBS (Figure 2) based on the absence of APTES signal on the regenerated SPR chip. The areal

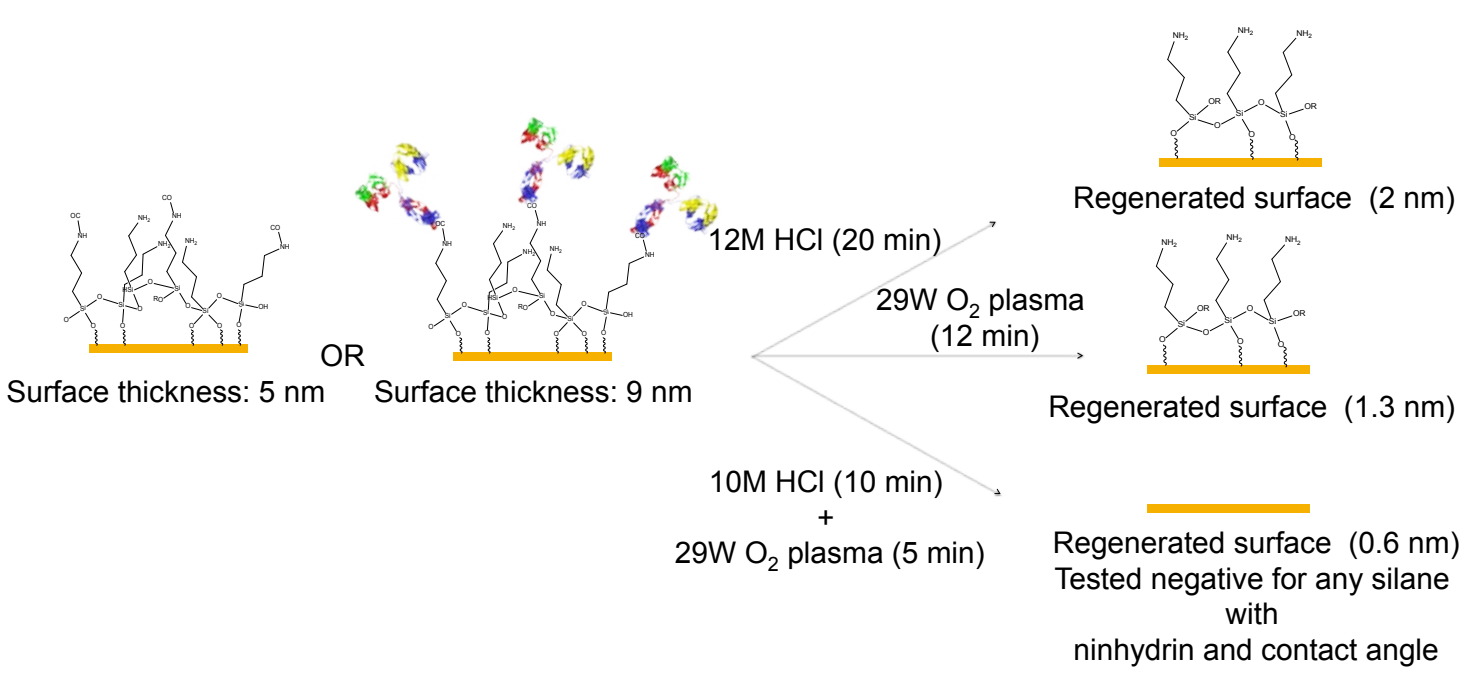

Figure 1: Schematic representation of complete regeneration of the silane-functionalized Au surface by acid and/ or plasmolysis of the siloxane bonds. The surface was characterized with ellipsometry and Rutherford back scattering (RBS) analysis for detection of any residual APTES on the surface. 


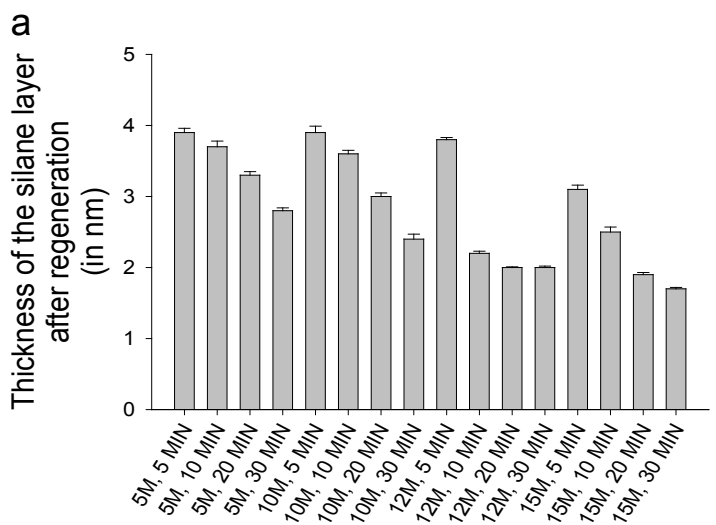

$\mathrm{HCl}$ acid strength with exposure time

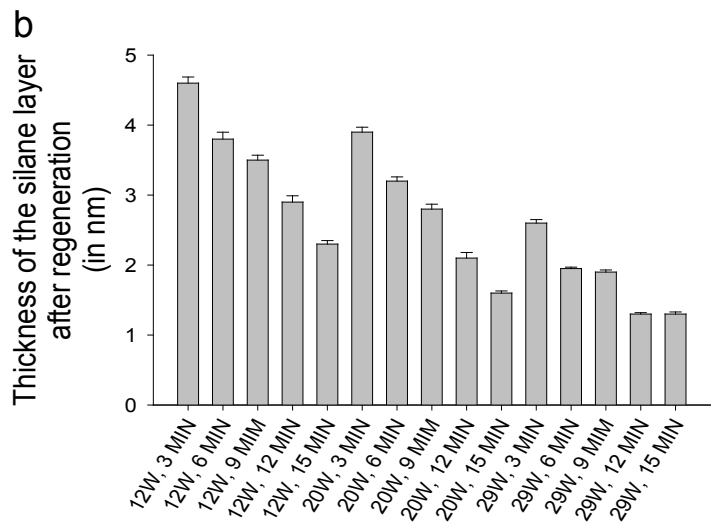

$\mathrm{O}_{2}$ Plasma strength and time of exposure

C

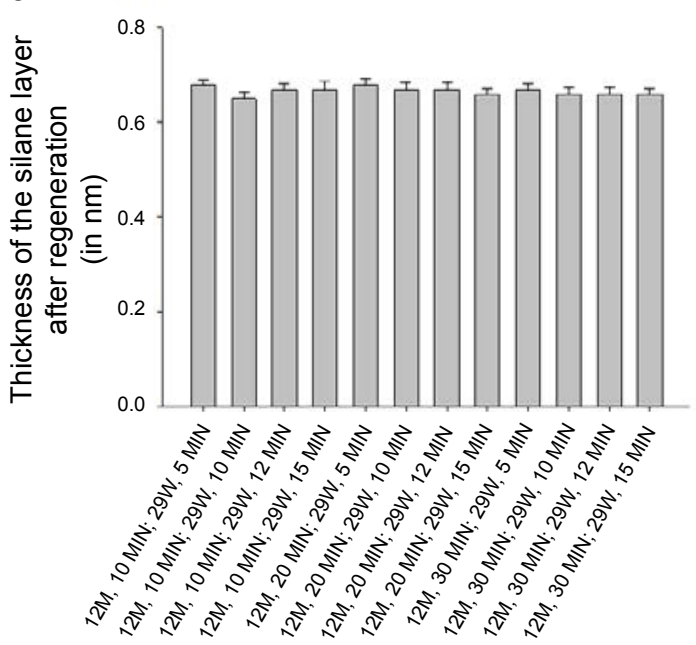

d

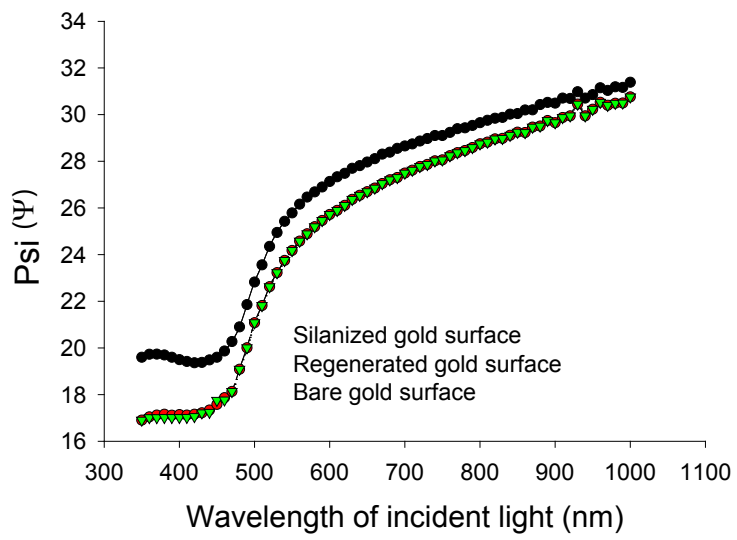

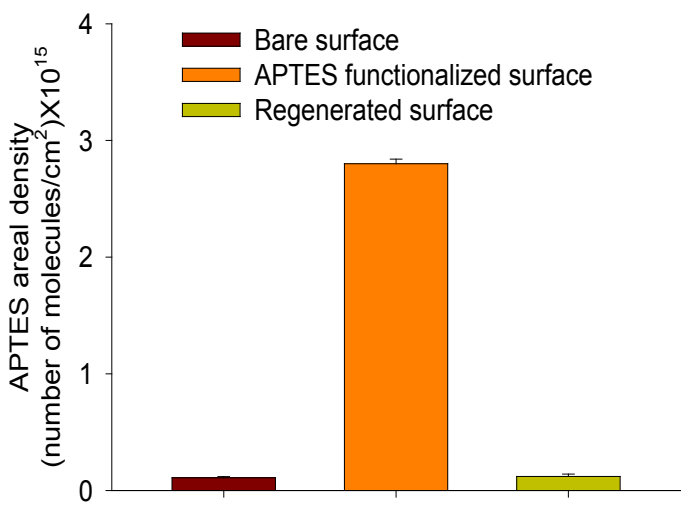

Figure 2: Optimization of surface regeneration procedure (a) siloxane bond lysis at various acid ( $\mathrm{HCl}),(\mathrm{b})$ various $\mathrm{O}_{2}$ plasma, and (c) their combined strengths as a function of exposure time ( $\mathrm{min}$ ). Thickness for each set was monitored using ellipsometry as depicted in'd'. (e) Evaluation of areal density of APTES on bare, APTES-functionalized and regenerated Au surfaces by Rutherford back scattering (RBS). Error bars are standard deviation.

density of APTES after APTES functionalization on the regenerated SPR Au chip was similar to that on the fresh SPR chip, which shows the complete regeneration of Au surface. During 40 consecutive HFA immunoassays, the areal density of APTES was highly-reproducible, which demonstrates that the developed regeneration procedure does not affect the surface properties of the Au layer.The developed Au surface regeneration procedure was optimized by determining the appropriate strength and treatment time of $\mathrm{HCl}$ and $\mathrm{O}_{2}$ plasma (individually in the initial stages, and in combination thereafter). Initially the efficiency of regeneration was assessed by treating with $5,10,12$ and $15 \mathrm{M}$ of $\mathrm{HCl}$ for 


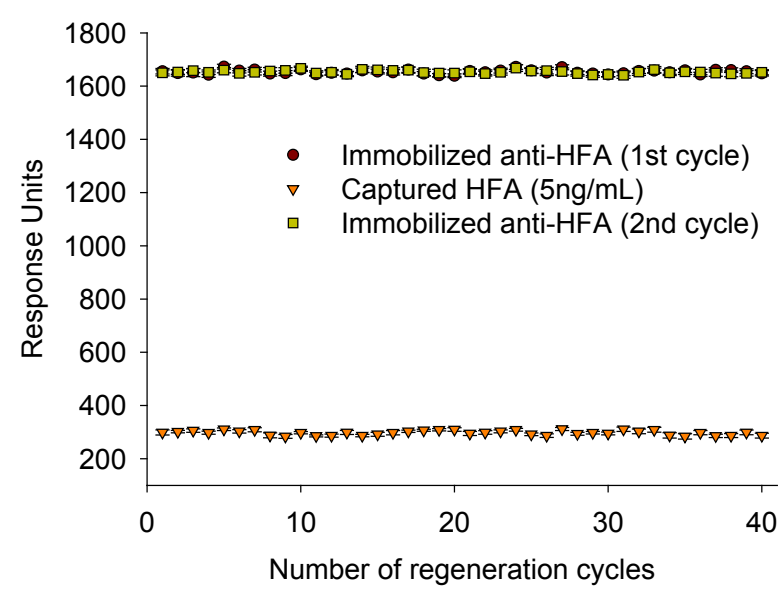

Figure 3: Reproducibility of antibody immobilization and antigen detection on the SPR Au chip, which was reused 40 times after regenerating the Au surface using the developed procedure. This process was mainly cycles of surface functionalization, assay performance, and regenerating the surface followed by repeating the cycle over 40 times. Error bars are \%CV.

varied duration i.e. 5, 10, 20 and $30 \mathrm{~min}$. The treatment with $15 \mathrm{M} \mathrm{HCl}$ for $30 \mathrm{~min}$ was found to be most appropriate for regenerating down to $1.7 \mathrm{~nm}$ thickness. However, the acid treatment does not regenerate the surface completely due to the role played by the respective anions generated by acid treatment in the dissolution of siloxane bonds [22]. These anions, such as hydroxyl $\left(\mathrm{OH}^{-}\right)$, sulfate $\left(\mathrm{SO}_{4}^{-2}\right)$ and chloride $\left(\mathrm{Cl}^{-}\right)$, potentially destabilize the siloxane frame work in the presence of water. However, the acidic environment enhances the polymerization of silanes, which is responsible for incomplete regeneration. Therefore, our initial acid-treatment approach was followed by $\mathrm{O}_{2}$-plasma scrapping/ etching [23].

The $\mathrm{O}_{2}$-plasma scrapping was done at plasma strength of 12, 20 and $29 \mathrm{~W}$ for $3,6,9,12$ and 15 min such that $29 \mathrm{~W} \mathrm{O}_{2}$ plasma treatment for $12 \mathrm{~min}$ was most effective and regenerated down to $1.3 \mathrm{~nm}$ thickness (Figure 2). The $\mathrm{O}_{2}$-plasma discharges are known to ionize the siloxane bonds present in the liquid phase to the silanol moieties in the vapour phase [23]. However, the same phenomenon might have occurred with the silane coated on the Au surface, as evident from the decrease in the layer thickness.

Finally, the surface regeneration was checked with various combinations of $\mathrm{HCl}$ and $\mathrm{O}_{2}$ plasma. For the initial $\mathrm{HCl}$ treatment step, $12 \mathrm{M}$ and $15 \mathrm{M} \mathrm{HCl}$ was used for 10,20 and $30 \mathrm{~min}$; whereas, for the $\mathrm{O}_{2}$ plasma scrapping, 29 $\mathrm{W} \mathrm{O}_{2}$ plasma was employed for 5, 10, 12 and 15 min. The complete regeneration of Au surface was obtained by treating it with $12 \mathrm{M} \mathrm{HCl}$ for $10 \mathrm{~min}$ followed by $\mathrm{O}_{2}$ plasma scrapping for $5 \mathrm{~min}$, which completely removed the APTES coating. This was confirmed by the absence of silicon peak due to APTES functionalization on the Au surface by RBS. A negative ninhydrin test and a contact angle of $28^{\circ}$ cross-validated the absence of silane on the surface.

The regenerated SPR Au chip was reused 40 times for SPR-based HFA immunoassay following the same bioanalytical procedure i.e. APTES-functionalization, anti-HFA antibody immobilization, HFA detection and surface regeneration. The reproducibility of APTES functionalization in each cycle was confirmed by ellipsometry and RBS, as discussed previously. The areal density of APTES after functionalization was consistently uniform in each detection cycle. The immobilization density of anti-HFA antibody and the subsequent detection of HFA was also highly reproducible in each cycle, as confirmed by the SPR response (Figure 3). The percentage coefficient of variance in APTES functionalization, anti-HFA immobilization and HFA detection, during 40 consecutive HFA immunoassays on the same SPR chip after regeneration, were 3-5, 4-6 and 2-4\%, respectively. Therefore, the developed $\mathrm{Au}$ surface regeneration procedure completely regenerates the Au surface without affecting its surface properties. It increased the cost-effectiveness of expensive SPR Au chip as the same SPR chip can be reused many times without any loss of activity. The developed regeneration is presently being investigated on other potential chemistries, based on caboxymethyl dextran, protein $\mathrm{A}$ and thiols, where it may yield similar results.

\section{Conclusion}

A simple procedure was devised for the complete regeneration of Au surface in the SPR Au chips that were used for HFA immunoassay. The SPR-based HFA immunoassay involved the functionalization of cleaned Au chip with APTES, followed by covalently binding of antiHFA antibody, blocking with $1 \%$ BSA, and detection of HFA. The regenerated SPR Au chip was effectively reused 40 times for HFA immunoassay with a very high reproducibility at each process step i.e. APTES functionalization, anti-HFA binding and HFA detection. The reproducible APTES functionalization of regenerated Au surface was confirmed by consistent areal density of APTES in RBS analysis, while the reproducible anti-HFA immobilization and HFA detection were determined by their SPR responses. The devised Au surface regeneration procedure will be immensely useful as it will enable the multiple reuse of costly SPR Au chip for cost-effective SPR-based immunoassays.

\section{Acknowledgement}

We acknowledge Bristol Myers Squibb (BMS), Syracuse, USA and the Industria Development Authority, Ireland, for the financial support under the CBAS project code 116294. We are thankful to Dr. Nam Cao Hoai Le of Biomedical Diagnostics Institute for valuable discussions pertaining to the analysis of ellipsometry data.

\section{References}

1. Loo L, Wu W, Shih WY, Shih WH, Borghaei H, et al. (2011) A rapid method to regenerate piezoelectric microcantilever sensors (PEMS). Sensors (Basel) 11 5520-5528.

2. Wang R, Lajevardi-Khosh A, Choi S, Chae J (2011) Regenerative Surface Plasmon Resonance (SPR) biosensor: Real-time measurement of fibrinogen in undiluted human serum using the competitive adsorption of proteins, Biosens Bioelectron 28:304.

3. Yuan YJ, Gopinath SCB, Kumar PKR (2011) Regeneration of commercia Biacore chips to analyze biomolecular interactions, Opt Engineer. 50: 034402

4. Drake AW, Klakamp SL (2011) A strategic and systematic approach for the determination of biosensor regeneration conditions. J Immunol Methods 371: 165-169.

5. Pei R, Cui X, Yang X, Wang E (2000) Real-time immunoassay of antibody activity in serum by surface plasmon resonance biosensor. Talanta 53: 481488.

6. Campbell GA, Mutharasan R (2006) Detection of Bacillus anthracis spores and a model protein using PEMC sensors in a flow cell at $1 \mathrm{~mL} / \mathrm{min}$. Biosens Bioelectron 22: 78-85.

7. Kausaite-Minkstimiene A, Ramanaviciene A, Kirlyte J, Ramanavicius A (2010) Comparative study of random and oriented antibody immobilization techniques on the binding capacity of immunosensor. Anal Chem 82: 6401-6408.

8. Ibii T, Kaieda M, Hatakeyama S, Shiotsuka H, Watanabe H, et al. (2010) Direct immobilization of gold-binding antibody fragments for immunosensor applications. Anal Chem 82: 4229-4235.

9. Renberg B, Shiroyama I, Engfeldt T, Nygren PK, Karlström AE (2005) Affibody 
Citation: Dixit CK (2014) Surface Regeneration of Gold-Coated Chip for Highly-Reproducible Surface Plasmon Resonance Immunoassays. J Biosens Bioelectron 5: 149. doi: 10.4172/2155-6210.1000149

Page 5 of 5

protein capture microarrays: synthesis and evaluation of random and directed immobilization of affibody molecules. Anal Biochem 341: 334-343

10. Herlem G, Segut O, Antoniou A, Achilleos C, Dupont D, Blondeau-Patissier $V$ (2008) Electrodeposition and characterization of silane thin films from 3-(aminopropyl) triethoxysilane, Surface and Coatings Technology 202: 1437.

11. Mitsyuk B (1984) Mechanism of silica dissolution and state of silicic acid in hydrothermal solutions, Theoretical and Experimental Chemistry 19: 554.

12. Vasant EF, Voort P, Vrancken K (1995) Studies in surface science and Catalysis. Characterization and Chemical Modification of the Silica Surface, Elsevier Science and Technology, Amsterdam.

13. Cypryk M, Apeloig Y(2002) Mechanism of the Acid-Catalyzed Si-O-Si Bond Cleavage in Siloxanes and Siloxanols. A Theoretical Study, Organometallics. 21: 2165 .

14. Castillo L, Barbosa S, Maiza P, Capiati N (2011) Surface modifications of talcs. Effects of inorganic and organic acid treatments, J Mater Sci 46: 2578.

15. Vashist SK, Dixit CK, MacCraith BD, O'Kennedy R (2011) Effect of antibody immobilization strategies on the analytical performance of a surface plasmon resonance-based immunoassay, Analyst 136: 4431.

16. Dixit CK, Vashist SK, MacCraith BD, O'Kennedy R (2011) Evaluation of apparent non-specific protein loss due to adsorption on sample tube surfaces and/or altered immunogenicity. Analyst 136: 1406-1411.

17. Spencer MJS, Nyberg GL (2004) Adsorption of silane and methylsilane on gold surfaces, Surf Sci 573: 151.

18. Jung A,Gronewold TMA, Tewes M, Quandt E, Berlin P (2007) Biofunctional structural design of SAW sensor chip surfaces in a microfluidic sensor system, Sensors Actuators B: Chem 124:46

19. TambolliS, Minunni M, Mascini, GL, Wang R (2004) Improved Plasmon resonance sensor for DNA sensing 42.

20. Siqueira Petri DF, Wenz G, Schunk P, Schimmel T (1999) An Improved Method for the Assembly of Amino-Terminated Monolayers on $\mathrm{SiO}_{2}$ and the Vapor Deposition of Gold Layers, Langmuir 15: 4520.

21. Smith T (1980)The hydrophilic nature of a clean gold surface, J.Colloid Interface Sci 75: 51.

22. Bai S, Urabe S, Okaue Y, Yokoyama T (2009) Acceleration effect of sulfate ion on the dissolution of amorphous silica. J Colloid Interface Sci 331: 551-554.

23. Leu GF, Brockhaus A, Engemann J (2003) Diagnostics of a hexamethyldisiloxane/oxygen deposition plasma, Surface and Coatings Technology 174-175. 